\title{
GW23-e0901 A STUDY OF GENE-GENE INTERACTION OF MHC2TA, VAMP8 AND HSP70-2 POLYMORPHISM WITH CORONARY HEART DISEASE
}

doi:10.1136/heartjnl-2012-302920d.9

Tian Ke-jun, Zhong Yi-ming. The First Affiliated Hospital of Ganzhou Medical University

Objectives To investigate the impact of Gene-gene interaction of MHC2TA-168A/G, VAMP8(rs1010), HSP70-2+1267A/G on the risk of coronary heart disease.

Methods A total of 185 coronary heart disease patients (male120, female 65, median age 64.57 \pm 10.94 ) were selected from Cardiovascular department of our hospital, Of these CHD patients 119 are myocardial infarction, others are angina pectoris patients. The control group is consisted of 149 subjects (male 96, female 53, median age 63.82-49.01), Human genome DNA was extracted by DNA extraction kit. The heat-shock protein 70-2 gene+1267A/G polymorphism, the vesicle-associated membrane protein 8 gene (rs1010) polymorphism were all detected by PCR-restriction fragment length polymorphism (PCR-RFLP) method. Major histocompability complex class II transactivator gene-168A/G polymorphism was all detected by misparing PCR-restriction fragment length polymorphism (mpPCR-RFLP) method. A multifactor dimensionality reduction analysis was carried out for interaction of genes by opening free MDR Software.

Results The MHC2TA gene-168A/G polymorphism, VAMP8 gene (rs 1010) polymorphism and HSP70-2 gene+1267A? G polymorphism also exist in this study population. The allelic distribution of the MHC2TA gene, the VAMP8 gene and the HSP70-2 gene in both $\mathrm{CHD}$ and control groups were in the Hardy-Weinberg equilibrium. The Interaction of MHC2TA-168A/G, VAMP8(rs1010) HSP70-2+1267A/G genetic polymorphisms was observed using Multifactor Dimensionality Reduction Analysis, OR of three genetic polymorphisms simultaneously was 5.21 times more than MHC2TA alone.

Conclusions The gene-gene interaction was observed among the polymorphism of MHC2TA-168A/G; VAMP8 (rslOlO) and HSP70$2+1267 \mathrm{~A} / \mathrm{G}$, three genetic polymorphisms simultaneously may increase the risk of Coronary heart disease obviously. 